\title{
GETTING AWAY FROM NUMBERS: USING QUALITATIVE OBSERVATION FOR AGENT-BASED MODELLING
}

\author{
LU YANG* $^{*}$ and NIGEL GILBERT ${ }^{\dagger}$ \\ Centre for Research in Social Simulation, \\ Department of Sociology, University of Surrey, \\ Guildford, Surrey, GU2 7XH, UK \\ *l.yang@surrey.ac.uk \\ ${ }^{\dagger}$ n.gilbert@surrey.ac.uk \\ Received \\ Revised
}

\begin{abstract}
Although in many social sciences there is a radical division between studies based on quantitative (e.g. statistical) and qualitative (e.g. ethnographic) methodologies and their associated epistemological commitments, agent-based simulation fits into neither camp, and should be capable of modelling both quantitative and qualitative data. Nevertheless, most agent-based models (ABMs) are founded on quantitative data. This paper explores some of the methodological and practical problems involved in basing an ABM on participant observation and proposes some advice for modellers.
\end{abstract}

Keywords: Agent-based models; qualitative data; ethnography.

The right question isn't: Does the number mean anything? The right question is: Does the number correspond to a difference that makes a difference in the kind of world being modelled. ${ }^{\text {a }}$

\section{Introduction}

Most agent-based models (ABMs) are intended to simulate some real-world phenomena and are therefore designed and validated by referring to data collected from the social world. Most often, such data are in numerical form. For example, the data may record the number of agents over time, obtained from organisational or government records; agents' opinions about some issue, derived from attitude surveys; or the number of transactions and their prices obtained from market records. There are well-developed statistical techniques for summarising such data and comparing them with the outputs of simulation models.

Unlike most other modelling approaches, there is nothing inherently quantitative about agent-based modelling. It should be as easy to develop and validate

although the best-known techniques make assumptions about the statistical distribution of the data that may not be appropriate for many ABMs, e.g. those that generate power-law distributions. 
$1 \quad$ ABMs with qualitative data as it is with quantitative data. Nevertheless, this is far less often done (a noteworthy exception is Agar [1,2]). In the next section, a

3 distinction is made between two types of qualitative data: one that can be used in almost the same way as quantitative data and one that for methodological reasons

5 must be treated quite differently. Section 3 briefly describes an example of building a model based on qualitative data, and Sec. 4 considers the extent to which

7 ethnographic data match the ABM methodology. Section 5 concludes with a few tentative guidelines for using such data for agent-based modelling.

\section{Qualitative Data and Ethnographic Research}

At its simplest, qualitative data cannot be converted into numeric values without distorting the information they contain. There are two types of such data and they are fundamentally different in nature. First, there are data that are collected using social measurement techniques such as surveys. The data may be gathered using nominal (e.g. black, white, green) or ordinal (e.g. agree, neutral, disagree) scales, rather than interval or ratio (i.e. numerical) scales [3], but, like conventional quantitative data, the measurements are made on variables with one value for each respondent and can be stored and analysed as a variable/actor matrix. Such qualitative data can be collected, managed and analysed using methods similar to those developed for quantitative data, with the exception of statistical techniques that require arithmetic operations on the values [4,5]. For instance, with a categorical scale such as "political party voted for in the last election", it would be inappropriate to calculate the mean vote, although it is still possible to find the modal (most frequent) party voted for. Because categorical and ordinal data can be used in the construction and validation of ABMs in much the same way as quantitative data, this type of data will not be further considered here.

The idea of qualitative data also encompasses an entirely different type of data (and the common use of the term "qualitative" to cover both often leads to confusion). These are data that are generally textual in form, such as field notes obtained from observation, transcripts of interviews, and published documents, although they can sometimes consist of images, video or audio. Such data are not readily converted into a variable/actor matrix without losing information or doing an injustice to the data. The important feature of such data is that they are about the meanings that participants ascribe to events and actors. To distinguish such data from the categorical data mentioned above, they will be called "ethnographic" data [6].

Ethnographic data are often collected and analysed on the basis of epistemological and ontological assumptions that are significantly different from those typically adopted by quantitative researchers. At first sight, these assumptions may seem to get in the way of their application to the design and validation of ABMs, but in fact the difficulties are not as great as they appear. The basis for most ethnographic 


\section{$\mathbf{1}_{\text {st Reading }}$}

March 26, $2008 \quad$ 19:8 WSPC/169-ACS $\quad 00155$

data collection ${ }^{\mathrm{b}}$ includes:

(1) Epistemological orientation: Ethnographic researchers often espouse naturalism - seeking to understand the particularities of a social phenomenon in its own terms in rich detail - while quantitative researchers often, although not always, adopt a covering law model of explanation seeking deductive laws that are of universal or near-universal application. Agent-based modellers tend to adopt a middle way, expecting their models to have some degree of generality, but rarely proposing that the models could have universal application (although some game theorists come close to that ambition).

(2) Causation: While quantitative research is generally concerned with identifying correlations between variables, ethnographic studies are more interested in causal mechanisms, i.e. "by what intermediate steps, a certain outcome follows from a set of initial conditions" [7].

(3) Ontological perspective: Ethnographic studies are frequently based on constructivism [8], which considers that social actors continually (re)construct their social world (rather than it being objectively available to them a priori). This contrasts with the positivist or, more frequently, realist orientation of most quantitative and agent-based modellers [9]. Critical realism [10] takes the approach that there are some "real" social processes or mechanisms existing that generate the observations that actors and researchers perceive.

(4) Methodological orientation: Ethnographic researchers generally adopt an inductive approach, in which theoretical categories are generated from consideration of the data (e.g. the procedure called "grounded theory" [11]). In contrast, quantitative research usually involves testing hypotheses and, at least in their writings, modellers tend to adopt a deductive approach, in which a simulation, designed on the basis of some prior theory, is tested to see whether it conforms to observed data.

(5) Importance of generalisation: Many ethnographic researchers adopt an idiographic stance, i.e. aiming to understand the particular and contingent character of the case they are studying, and tend to be sceptical about the possibility and the value of generalisation to universals. Agent-based modellers often assume that their models express some general patterns and structures, although they will be instantiated with particular parameters to represent some specific case. ${ }^{\mathrm{c}}$

(6) Emic versus etic orientation: An emic description is one that is formulated in terms that are meaningful to the actor [13]. Typically, ethnographic data are emic, in contrast to etic data, which are formulated using concepts meaningful to the researcher, but not necessarily to the actor. Both quantitative

\footnotetext{
${ }^{\mathrm{b}}$ Inevitably, not all ethnographic, quantitative and agent-based studies exactly match the descriptions suggested in this list, but the majority do.

"For example, a "history-friendly" model [12].
} 
Table 1. Characterisation of three types of social research.

\begin{tabular}{|c|c|c|c|}
\hline & Quantitative & Agent-Based & Qualitative \\
\hline $\begin{array}{l}\text { Epistemological } \\
\text { orientation }\end{array}$ & $\begin{array}{l}\text { Deductive-statistical } \\
\text { laws }\end{array}$ & & Inductive laws \\
\hline Causation & Correlational & Mechanism & Process \\
\hline Ontology & Positivist & Realist & Constructivist \\
\hline Methodology & Hypothesis testing & Model validation & Grounded theory \\
\hline Generalisation & Seeks general laws & $\begin{array}{l}\text { Seeks middle range } \\
\text { models }[15]\end{array}$ & Idiographic \\
\hline Emic/etic & Etic & Etic & Emic \\
\hline $\begin{array}{l}\text { Temporal and } \\
\text { context emphasis }\end{array}$ & $\begin{array}{l}\text { Cross-sectional and } \\
\text { context-free }\end{array}$ & Time-important & Context-sensitive \\
\hline
\end{tabular}

theories and ABMs often use concepts (e.g. power law, evolution, utility) that are unlikely to be meaningful to those whose behaviour is being modelled. ${ }^{\mathrm{d}}$

(7) Process and context: There is a strong emphasis in most ethnographic research on the process, i.e. on how and why things change over time. Mainly because it is often difficult or expensive to collect time-varying quantitative data, there is less emphasis on the process in most quantitative studies. In this respect, agentbased modelling, in which the passage of time is represented by simulated time steps, is closer to ethnographic than to quantitative research. Ethnography also emphasises context: that the meaning of actions depends on the context in which they are carried out. This is also a usual element in ABMs, where agents are given rules of behaviour that are context-specific (i.e. whether and how a rule is activated depends on the current state of the agent and its environment).

These similarities and differences between agent-based modelling and ethnographic research are summarised in Table 1 and illustrated in the next section, which describes research on the socialisation of members into service work teams.

\section{An Example}

Lu Yang has been conducting a study of the socialisation of newcomers into existing organisational groups, and examining how the entry of a new member reshapes the members' interactions and the structure of the group [16]. Data were gathered by participant observation of two service work groups: one in a library, where the group task was to record book loans and returns, stack returned books and perform routine administration; and the other in a nursing home, where the group task was to carry out personal care for a number of bedridden patients. Each observation was conducted for a period of six months by participation in the work group as a new team member. The study generated copious and detailed field notes recording the

${ }^{\mathrm{d}}$ But this is not always true: see the companion modelling movement [14] and participatory modelling more generally. 


\section{$\mathbf{1}_{\text {st Reading }}$}

March 26, $2008 \quad$ 19:8 WSPC/169-ACS $\quad 00155$

researcher's observations and providing direct evidence of the activities of members and changes in group behaviour.

The "translation" of these field notes into a rule-based computer program involved two stages. First, a narrative account of the socialisation process was constructed. This began with an overall picture of the setting, the culture and the members' everyday procedures. For example, the nursing home was situated in the countryside of south-east England. It is privately owned, medium in size, and the residents there are old and with various clinical needs. The functioning of the nursing home, including details such as how many nurses and carers were employed, what kind of work they engaged in and the procedures they used, was described.

Then, a number of themes were identified, and illustrated with stories about specific events and incidents that were observed. The observation data were used to discuss, for instance, the high employee turnover rate, variations in the quality of service, the boundaries of friendship groups, the hostility of existing staff towards newcomers, etc. Explanations of these features of life in the nursing home were formulated in terms of themes, e.g. that the care workers established their identities partly by reference to their ethnicity, that co-workers often got angry with others due to task conflicts, and that new carers learnt practical skills not from formal training but from working with more experienced carers.

Much ethnographic research stops at this point, with a plausible narrative that aims to explain the observations by reference to descriptions of social processes. However, such descriptions tend to be vague and difficult to validate. We wished to take the analysis a stage further by constructing an ABM that represented abstract versions of these processes.

The second stage of the analysis therefore consisted of the design and implementation of an ABM that encapsulated some of the essential features of socialisation observed in the two settings. As recommended by Dey [17], qualitative data analysis "involves a process of abstracting from the immense detail and complexity of our data those features which are most salient for our purpose". The ABM holds the promise of helping to identify which features are most salient, ensuring that the extracted features are consistent and coherent, and providing a way of validating our conclusions by running the simulation. A recurring problem, however, was how to model aspects of the settings without making them more precise or concrete than the observational data would warrant (cf. Whitehead's "Fallacy of Misplaced Concreteness" [18]).

The model, implemented in NetLogo [19], was designed to have the following features:

(1) Agents work on tasks. Agents and tasks are each represented as objects (i.e. as "turtles" in NetLogo). A decision had to be made about how many of each type of object to create. For example, this involved "abstracting" from the number of carers that were employed in the nursing home (the actual number varied from one week to the next and by shift) to a constant number of agents (that 
could varied from 1 to 9 between runs) and categorising the activities of the carers into precisely 10 tasks.

(2) Agents have attributes. It was observed that people's demographic characteristics (e.g. age, gender, race and "aggressiveness") were salient in their relationships with other team members. In the model, each characteristic is represented by the value of an agent attribute. The attributes are used to calculate the initial attraction that each agent has to the others. There are many social-psychological studies that support the proposition that demographic attributes affect social integration (e.g. [20-22]). However, neither these studies nor our observations are able to quantify the degree of attraction given by these attributes, nor do they say anything about the attributes' relative importance or the functional form of the relationship. Consequently, an arbitrary choice was made (a function based on the Pearson product-moment correlation coefficient) and a sensitivity analysis conducted to see whether variations made a substantial difference to the outcomes (they did not).

(3) Tasks have a priority level and a completion time. Since there are a maximum of 10 tasks at any time, the priority level ranges from 0 to 9 . When applied to the library service team, priority level 9 represents front-line service; priority 8 means sorting book trolleys, and so on. The rank ordering of priority was based on what had been learned from observation of library work. The time taken to complete each task and the increase in speed at which a task is performed when more than one person works at it were also estimated from the observations. However, while in the model all tasks were assumed to take the same amount of effort, in reality different tasks took different times and the rates at which they were carried out depended on many factors beyond the number of workers assigned to them.

(4) Agents that leave are immediately replaced by new, unsocialised agents. Observation confirmed that a new employee will be recruited to replace a worker who leaves. For simplicity, the model was designed so that one worker left and was replaced after a fixed number of time steps; in the settings, the timing of resignations was irregular.

(5) Agents have "emotions". The field notes showed that emotion played a key role in the socialisation of new team members in both settings. A typical instance is shown in Fig. 1, an extract from the observer's notes.

This was represented in the model by formulating rules of interaction such that if any one agent displays certain emotions, other agents check their attraction value towards this agent. If an agent decides to respond to the one who displays emotion, the attraction matrix is updated. Unfortunately, however, it is not clear how to decide on how much the attraction value should be increased. Nor is it easy to settle on a value for the emotion display threshold. There is no theory or empirical basis for these and other numerical manipulations. 


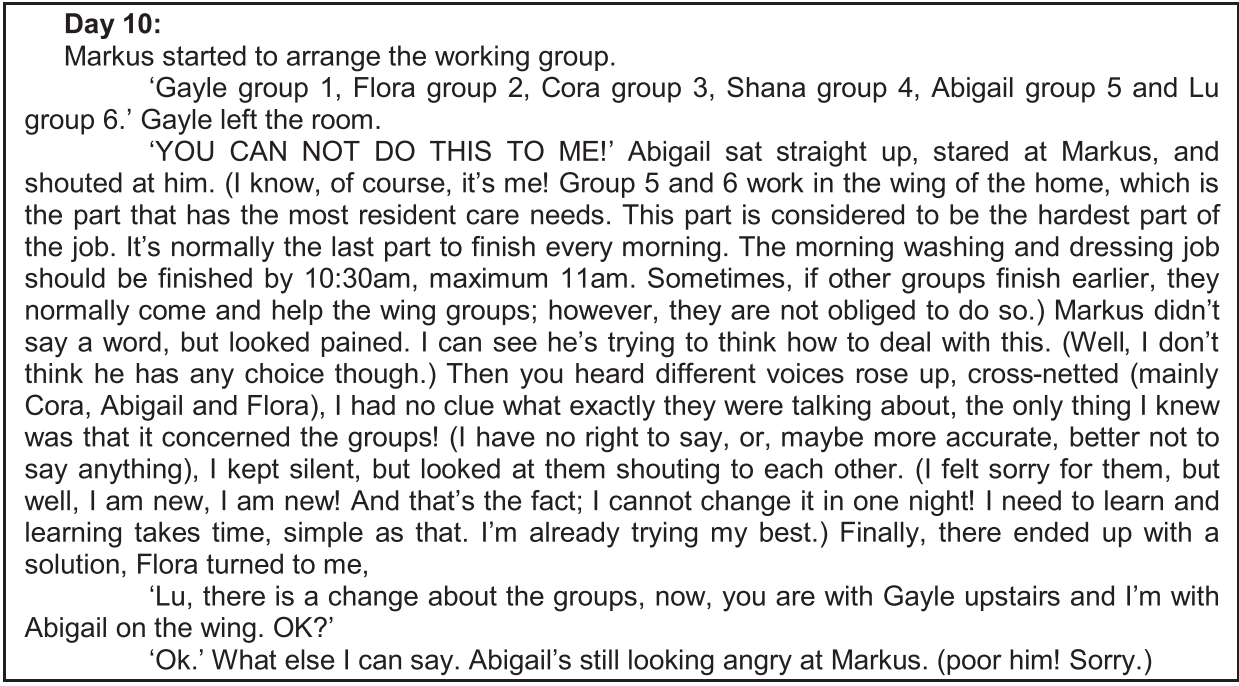

Fig. 1. Extract from field notes recorded on the tenth day of participant observation at the nursing home. [All the participants' names have been changed except for the observer's (Lu).]

Despite our efforts to reduce the number of unsupported numerical constants and arithmetical operations within the model, it did not prove possible to eliminate many of them. One could "cheat", removing numbers by tricks of coding, or increase the apparent quantification by using numerical instead of symbolic values for all constants. While the presence of unjustifiable, "magic" constants in the code was the clearest example, it was not the only instance where the model had to be made more definite than the ethnographic record could justify. For example, in the model, agents that leave are immediately replaced (i.e. in the next time step). It looks as though there is no quantification here, but there is an implicit zero number of time steps between one agent leaving and the next arriving. In practice, in both the library and the nursing home, there was almost always some time gap before a new member of staff could be recruited, trained and formally inducted.

To resolve this problem, it was necessary to engage in a further process of abstraction. As noted above, both ABM modellers and ethnographic researchers are interested in mechanisms, i.e. the processes or sequences of events that causally link initial conditions and outcomes [7,23]. A mechanism is a theoretical construction described in abstract terms (for example, the mechanism of an epidemic involves a virus infecting a susceptible victim, who in turn infects another victim - here the concepts "virus" and "victim" are theoretical terms). The ethnographer observes an instantiation of the mechanism (Ann infects Bob, or, in the nursing home, Charles has an argument with David) and infers the mechanism from his or her observations, through a process of abstraction. In doing so, many quantities, even if they are observed at all, are discarded (for example, the account of the epidemic may 
ignore the time taken for the virus to make the victim infectious; in the library, the ethnographer may record but then disregard the number of arguments between Charles and David and simply note the existence of conflict). The modeller has to go the other way: given an abstract social process or mechanism, he or she has to invent some numbers in order to fill out the theory sufficiently to create a working implementation. The important issue in inventing these numbers is not their specific values, but that the implemented model must be an instantiation of the desired mechanism.

\section{The Relationship Between ABMs and Ethnographic Data}

The purpose of building an ABM to represent the library and nursing home case studies was to formalise and verify the set of mechanisms that led to the main features of the settings that were observed. This involved creating a specification of the model and the behavioural rules to be followed by the agents, implementing and running the model, and comparing the outcomes with the ethnographic record. In terms of the issues listed in Table 1 of Sec. 2, this requires:

(1) A realist orientation, in which observable social action is considered to be generated by the modelled social processes. Although critical realism is usually associated with an objectivist stance, it is possible to couple realism with constructivism if one allows for agents to construct the meanings and concepts they use as a response to the social environment in which they act. However, given the fairly primitive models of cognition and language use in almost all current ABMs, modelling of such aspects is unusual.

(2) Either a deductive approach, in which the model is formulated and then tested against the ethnographic data, or an abductive one, in which a theory and thus a model is induced from the data and then the model is tested against the data and discrepancies used to improve the model.

(3) A concern with generalisation. ABMs normally represent a general theory about how some social phenomenon works. Ethnographic data about one particular setting can be used as a case study or a "critical case" that can be used to help to validate a model - more precisely, to falsify the model if it is incapable of generating a representation of the behaviour observed in the setting.

(4) An etic approach, i.e. one that uses the analyst's concepts, but where actors' concepts are, as far as possible, allowed to emerge from the operation of the model.

(5) The usual interest in process and context that can be found in most ABMs.

Working within these constraints, it seems possible and useful to construct an ABM that represents the processes or mechanisms that have been discovered from ethnographic research. However, the model cannot be expected to confine itself to representing the ethnographic data and no more, for an $\mathrm{ABM}$ will inevitably require many "numbers" or, more accurately, modelling assumptions, that are not to be 

at some abstract level the same mechanisms as are identified in the ethnographic analysis.

\section{Conclusions}

It has been suggested that it is possible to use ethnographic data, such as field notes from participant observation in the design and validation of ABMs, but that there are a number of areas where a modeller will find himself or herself having to move away from the conventional assumptions and methods of ethnographic research. While the agent-based modelling community needs to gain more experience of using ethnographies in their work, some advice can already be formulated for those contemplating using ethnographic data:

(1) Ethnographic data are usually very rich and detailed, while simulation models tend to be somewhat abstract. In order to bridge the gap, it will be necessary to develop analyses of the ethnographic data, possibly using computer-aided qualitative analysis (CAQDAS) tools ${ }^{\mathrm{e}}$ to help manage, code and search the textual data.

(2) Although typically an ethnographic study describes just one particular setting, it can be used as a case study of a more general class of settings. It may be useful either to conduct more than one ethnographic study, so that settings can be compared, or to locate descriptions of other settings that can substitute for first-hand data collection.

(3) Ethnographic data are often good sources of information about relationships, among actors and between actors and objects. A model of the data will need to represent such relationships, i.e. the model is likely to be based on networks of agents.

(4) Ethnographies usually contain descriptions of social processes that are equivalent to the kinds of mechanisms that are typically modelled by ABMs. This helps to lay out these processes explicitly in the ethnographic analysis before attempting to model them.

(5) The modeller will have to add "concreteness" to the model beyond the evidence in the data (e.g. choosing numeric values for thresholds and multipliers; selecting distributions for random number generators; assigning attributes to agents either at random or in some arbitrary way). This is an inevitable part of the process of using ethnographic data as the foundation of an ABM. As far as possible, such choices should be justified by conducting a sensitivity analysis and showing that they do not have a significant effect on the outcomes of the simulation, while recognising that it is impracticable to test the sensitivity of every aspect of a model.

esee, for example, the reviews and resources available at http://caqdas.soc.surrey.ac.uk 
1 (6) While a great deal of ethnographic data concern the observation of individual action, some structural and group data can also be gathered (e.g. who talks to whom and when; what collective events occur; who is involved in rituals). These "macro" features of the setting are valuable for the modeller. The model may generate outcomes and patterns that correspond to these features, and if it does, this helps to validate the model.

(7) The modeller should consider the implications of a stance that proposes that the way people structure their worlds is not based on an external reality but a culturally generated and fluid categorisation that is self-organising and emergent. While this constructivist perspective is hard to accommodate, given the state of the art in agent-based modelling, one can at least try to represent some of its implications.

Ethnographic data, we contend, are no less relevant to building ABMs than quantitative data. However, while there is already much experience in using quantitative data to design and validate $\mathrm{ABMs}$, there are relatively few examples of ABMs based on ethnographies and little advice to guide the modeller. We hope that this paper will inspire more consideration of how ethnographic data can best be used by modellers.

\section{Acknowledgment}

We acknowledge the partial support from the project "EMIL: EMergence In the Loop: simulating the two-way dynamics of norm innovation", funded by the European Commission Sixth Framework Programme - Information Society and Technologies - Citizens and Governance in the Knowledge-Based Society.

\section{References}

[1] Agar, M., My kingdom for a function: modeling misadventures of the innumerate, Journal of Artificial Societies and Social Simulation 6 (2003) 8.

[2] Agar, M., Agents in living color: towards emic agent-based models, Journal of Artificial Societies and Social Simulation 8 (2005) 4.

[3] Sirkin, R. M., Statistics for the Social Sciences (Sage, Thousand Oaks, California, 1995).

[4] Babbie, E., The Practice of Social Research (Wadsworth, 2004).

[5] Stevens, S. S., On the theory of scales of measurement, Science 103 (1946) 677-680.

[6] Agar, M., The Professional Stranger: An Informal Introduction to Ethnography (Academic Press, 1996).

[7] Mayntz, R., Mechanisms in the analysis of macro-social phenomena, Philosophy of the Social Sciences 34 (2004) 237-259.

[8] Berger, P. L. and Luckmann, T., The Social Construction of Reality: A Treatise in the Sociology of Knowledge (Anchor, 1967).

[9] Gilbert, N. and Chattoe, E., Hunting the unicorn: an exploration of the simulation of small group leadership. In: Saam, N.J. and Schmidt, B. (eds.) Cooperative Agents: Applications in the Social Sciences (Kluwer, Dordrecht, 2001), pp. 109-124. 
[10] Archer, M., Realist Social Theory: The Morphogenetic Approach (Cambridge University Press, 1995).

[11] Glaser, B., Basics of Grounded Theory Analysis (Sociology Press, Mill Valley, California, 1992).

[12] Malerba, F., Nelson, R., Orsenigo, L. and Winter, S., History-friendly models: an overview of the case of the computer industry, Journal of Artificial Societies and Social Simulation 4 (2001) 6.

[13] Headland, T., Pike, K. and Harris, M., Emics and Etics: The Insider/Outsider Debate (Sage, 1990).

[14] Barreteau, O., Our companion modelling approach, Journal of Artificial Societies and Social Simulation 6 (2003).

[15] Merton, R. K., Social Theory and Social Structure (Free Press, New York, 1968).

[16] Yang, L. and Gilbert, N., Case-Based Model of Emotional Expression Influence on Work Group Socialization and Performance (1st World Congress on Social Simulation, Kyoto, Japan, 2006).

[17] Dey, I., Qualitative Data Analysis: A User-Friendly Guide for Social Scientists (Routledge, London, 1993).

[18] Whitehead, A. N., Science and the Modern World (Free Press (Simon \& Schuster), 1925).

[19] Wilensky, U., NetLogo (Center for Connected Learning and Computer-Based Modeling, Northwestern University, Evanston, Illinois, 1999).

[20] Hoffman, E., The effect of race-ratio composition on the frequency of organizational communication, Social Psychology Quarterly 48 (1985) 17-26.

[21] McPherson, M., Smith-Lovin, L. and Cook, J. M., Birds of a feather: homophily in social networks, Annual Review of Sociology 27 (2001) 415-444.

[22] Ward, R.A., La Gory, M. and Sherman, S. R., Neighborhood and network age concentration: does age homogeneity matter? Social Psychology Quarterly 48 (1985) $138-149$

[23] Hedström, P. and Swedberg, R. (eds.), Social Mechanisms: An Analytical Approach to Social Theory (Cambridge University Press, 1998). 